\title{
Lidil
}

Revue de linguistique et de didactique des langues

$59 \mid 2019$

Prononcer les langues : variations, émotions, médiations

\section{L'accentuation en cours d'espagnol langue étrangère : apports de la phonétique expérimentale à l'évaluation de la production et de la perception de la prononciation}

Spanish Lexical Stress in the Teaching of Spanish As a Second Language:

Contributions of Experimental Phonetics to Assess Pronunciation Perception and Production

\section{Margarita Muñoz García}

\section{OpenEdition}

Journals

Édition électronique

URL : http://journals.openedition.org/lidil/6251

DOI : $10.4000 /$ lidil.6251

ISSN : $1960-6052$

Éditeur

UGA Éditions/Université Grenoble Alpes

Édition imprimée

ISBN : 978-2-37747-090-7

ISSN : 1146-6480

Référence électronique

Margarita Muñoz García, «L'accentuation en cours d'espagnol langue étrangère : apports de la

phonétique expérimentale à l'évaluation de la production et de la perception de la prononciation », Lidil [En ligne], 59 | 2019, mis en ligne le 01 mai 2019, consulté le 04 mai 2019. URL : http:// journals.openedition.org/lidil/6251 ; DOI : 10.4000/lidil.6251

Ce document a été généré automatiquement le 4 mai 2019.

(C) Lidil 


\section{L'accentuation en cours d'espagnol langue étrangère : apports de la phonétique expérimentale à l'évaluation de la production et de la perception de la prononciation}

Spanish Lexical Stress in the Teaching of Spanish As a Second Language: Contributions of Experimental Phonetics to Assess Pronunciation Perception and Production

Margarita Muñoz García

\section{Introduction}

Notre travail se situe dans le cadre de l'apprentissage de l'espagnol langue étrangère (désormais ELE) par des apprenants francophones. En général, la plupart des enseignants de langues s'accordent sur l'importance de la compétence orale dans l'apprentissage d'une langue étrangère ou seconde (L2) et l'une des priorités des apprenants est d'apprendre à communiquer oralement dans la L2. Du point de vue de l'apprentissage de la prononciation, lors de l'acquisition phonique d'une langue, on doit tenir compte aussi bien de l'acquisition des éléments segmentaux que des éléments suprasegmentaux (accentuation, rythme, intonation et pauses). Lorsqu'on parle, les mots sont intégrés dans la chaine parlée, accentués les uns par rapport aux autres. Certains mots marquent des frontières, d'autres portent une intonation particulière selon le contexte et l'intention du locuteur. En somme, la «prononciation est production phonique» (Cortés, 2002, p. 66). Lorsque les apprenants francophones se voient confrontés à l'ELE, ils vont devoir construire un nouveau "paysage sonore ", différent de celui de leur langue première (désormais L1) (Lhote, 1995, p. 22), avec de nouveaux sons mais également avec un autre 
rythme, une autre mélodie. D'où l'importance de prendre en compte toutes les composantes de la prononciation tant dans son enseignement qu'au niveau de la recherche.

Il a été souvent souligné que l'enseignement de la prononciation fait partie des éléments les plus délaissés dans l'apprentissage des langues (Spalacci, 2014, p. 176; Herry-Benit \& Pillot-Loiseau, 2012, p. 62) et plus particulièrement l'enseignement de la prosodie (Frost, 2010, p. 38). Un enseignant se rend vite compte que les francophones éprouvent des difficultés avec l'accentuation en espagnol à l'oral (Gil Fernández, 2007, p. 288). Cependant, dans certains cas, les francophones perçoivent ou produisent l'accent sans difficulté mais, dans d'autres cas, les difficultés sont évidentes et parfois persistent. L'enseignant peut donc se poser de nombreuses questions: Quelles difficultés ont-ils exactement? Pour quel type d'accentuation? Dans quels contextes? Nous avons adopté, dans notre travail, une approche expérimentale afin d'apporter des réponses concrètes à notre expérience d'enseignement. Nous avons souhaité évaluer les besoins spécifiques des apprenants francophones qui se confrontent à l'apprentissage de l'accentuation espagnole. Dans l'acquisition de la L2, la perception et la production phoniques sont des processus étroitement liés. La façon dont un processus influe sur l'autre est longuement étudiée depuis des décennies (Blanco Canales, 2016, p. 2). Certains auteurs préconisent un enseignement de la prononciation ciblant, autant que possible, à la fois perception et production (Herment, 2018, p. 75). Notre étude s'est donnée pour objectif d'étudier les phénomènes de perception et de production de l'accent lexical de la part d'apprenants francophones (Muñoz García, 2010, p. 173) en tenant compte des caractéristiques de deux langues et des différences d'accentuation. En espagnol, langue à accent partiellement libre, l'accent peut survenir sur l'une des quatre dernières syllabes du mot et possède une fonction distinctive qui agit sur l'axe paradigmatique (Quilis, 1993, p. 388-390 ; Hidalgo Navarro \& Quilis Merin, 2004, p. 230). Le français possède un accent primaire fixe oxyton qui frappe toujours la dernière syllabe du mot, du syntagme ou de la phrase. Il s'agit d'un accent de groupe et non d'un accent de mot (Di Cristo, 1981, p. 32) avec une fonction démarcative qui facilite le décodage des unités de sens ou syntagmes (Léon, 1992, p. 109). En outre, les processus de perception et de production de la parole sont des phénomènes complexes qui paraissent être en relation étroite avec les caractéristiques phonologiques de la langue d'un individu (Sebastián-Gallés, Bosch \& Costa, 1999, p. 53-88). En fonction des caractéristiques de deux langues et selon la tâche à réaliser, nous observerons quelle est l'influence de leur L1, le français, dans la perception et production de l'accent lexical de l'espagnol.

\section{Hypothèses}

Le but principal de cette étude est d'étudier de quelle façon les apprenants francophones perçoivent et produisent l'accent lexical de l'espagnol. Nous voulons examiner également quelles différences peuvent émerger entre la tâche de perception et la tâche de production. Pour ce faire, nous avons mis en place des tests de perception auditive ${ }^{1}$ et de production orale afin d'étudier de quelle façon la L1 des apprenants francophones de l'étude agit sur la perception de l'accent lexical espagnol et quelle relation s'établit entre leur perception et leur production. Ainsi, le test expérimental, que nous présentons ensuite, tente de répondre à ces questions afin de vérifier chacune des hypothèses qui $\mathrm{y}$ sont associées. Nous avons établi une hypothèse générale et plusieurs hypothèses 
particulières en fonction de variables prises en considération. Nous mesurons les pourcentages de bonnes réponses (BR) afin de tester l'incidence de la L1 dans la perception et la production de l'accent en espagnol langue étrangère.

En ce qui concerne la perception, une bonne réponse correspond à l'identification de la syllabe accentuée par les sujets comme la syllabe orthoépiquement accentuée: par exemple, « 'pú » comme la syllabe accentuée dans le mot proparoxyton 'público, lorsque les sujets signalent celle-ci sur la grille de réponses.

Une bonne réponse en production est réalisée lorsque l'accent est perçu par l'évaluateur sur la syllabe qui correspond à la syllabe accentuée du mot. Par exemple, dans le mot paroxyton to'mate, la BR est donnée lorsque l'évaluateur perçoit l'accent sur la deuxième syllabe, « 'ma».

\subsection{Hypothèse générale}

6 H0: Nous nous demandons si les performances des sujets seront similaires lors du traitement des stimuli en perception ou en production.

7 Compte tenu des résultats des expériences précédentes (Munoz Garcia, 2010, p. 189) et d'autres études portant sur la question (Mora, Courtois \& Cavé, 1997, p. 81 ; Schwab \& Llisterri, 2010, p. 320), nous pensons que la perception de l'accent lexical espagnol par des francophones sera globalement bonne et atteindra des pourcentages de BR assez élevés dans toutes les conditions.

8 L'influence de la L1 sur les productions en L2 a souvent été démontrée tant au niveau segmental que suprasegmental (Archibald, 1997, p. 277-278, dans Rasier, 2003, p.81; Cortés, 2002, p. 53 ; Rasier \& Hiligsmann, 2007, p. 59). La tendance des francophones à placer l'accent sur la dernière syllabe du mot a souvent été soulignée dans la littérature (Iruela, 2004, p. 41 ; Gil, 2007, p. 288). Enfin, dans notre pratique d'enseignante d'ELE à des francophones, nous constatons la tendance des apprenants francophones à déplacer l'accent sur la dernière syllabe. Nous pensons en conséquence que leurs productions seront influencées par le français et que nous retrouverons des productions orales avec un déplacement de l'accent sur la dernière syllabe.

Notre hypothèse générale présume donc que l'influence de la L1 sera plus marquée au niveau de la production qu'au niveau de la perception. De ce fait, les performances des sujets francophones, en tâche de production, seront inférieures à celles obtenues en perception et nous présumons des différences significatives entre la perception et la production.

\subsection{Hypothèses particulières}

\subsubsection{Effet du niveau des sujets}

10 Nous postulons que plus le sujet a un niveau de langue élevé, moins il aura de difficultés à percevoir l'accent lexical de l'espagnol. Des résultats obtenus lors des expériences précédentes ont montré que le niveau d'expertise de l'espagnol joue un rôle positif lors de la perception de l'accent lexical espagnol par des francophones (Muñoz Garcia, 2010, p. 190). 
11 Au niveau de la production, des travaux suggèrent que les apprenants d'une LE traversent différents stades d'interlangue (ex. d'apprenants néerlandais du français, Rasier, 2003, p. 81-83). Nous présumons donc que les pourcentages de BR augmenteront en fonction du niveau de langue des sujets et reflèteront une meilleure maitrise de l'accentuation.

\subsubsection{Effet du type d'intonation}

12 Les résultats des études précédentes en perception (Muñoz Garcia, Panissal, Billières \& Baqué, 2009, p. 494 ; Muñoz Garcia, 2010, p.163-164) ont révélé que les apprenants francophones montraient plus de difficultés à percevoir l'accent lexical dans les phrases en intonation montante que dans celles avec une intonation descendante ${ }^{2}$. Nous postulons que ce type d'intonation peut rendre la tâche de perception plus difficile et nous nous demandons si cette difficulté sera la même en tâche de production. Nous testons cette hypothèse en trois conditions : mots isolés, phrases en intonation montante et phrases en intonation descendante

\subsubsection{Effet du nombre de syllabes}

13 Le crible accentuel sera plus tenace en fonction du nombre de syllabes des mots à percevoir ou à produire: plus le mot contient de syllabes plus la perception ou la production de l'accent lexical peut s'avérer difficile. Tout comme pour l'effet de mots isolés ou en contexte, l'augmentation du nombre de syllabes rend la tâche cognitive plus difficile. Les résultats de Lafrance (2001, p. 112-113) vont dans ce même sens.

\subsubsection{Effet du type d'accentuation}

14 En fonction de l'endroit où l'accent se trouve dans les mots, on peut différencier en espagnol trois types de mots dans les formes non composées (Quilis, 1993, p. 389-390) : a) mots oxytons : la syllabe accentuée est la dernière du mot comme (ca'fé ou pelo'tón); b) mots paroxytons : la syllabe accentuée est l'avant-dernière du mot (co'media ou 'salto); c) mots proparoxytons : la syllabe accentuée est l'antépénultième du mot ('música ou 'plátano). Nous nous demandons s'il existe des degrés de difficulté différents en perception et en production en fonction du type d'accentuation.

En raison de la fréquence globale dans la langue, il est permis de présumer que les sujets obtiennent les meilleures performances pour les mots paroxytons, puisqu'approximativement $80 \%$ des mots espagnols sont paroxytons dans la langue parlée (Quilis, 1981, p. 333-336). Nous présumons que les sujets auront davantage de difficultés avec l'accentuation oxytone tant en perception qu'en production. Nous pensons que la similarité peut poser d'avantage des difficultés, comme il s'est avéré lors de prétendantes études en perception (Muñoz Garcia, 2010, p. 164-166).

\subsubsection{Effet de la transparence lexicale}

16 Notre hypothèse présume que les apprenants francophones percevront et produiront plus difficilement l'accentuation des mots dits transparents que les mots non transparents. La ressemblance segmentale phonétique entre les mots français et espagnols peut être une source de difficulté. Nous émettons donc la même hypothèse que pour la similarité concernant l'accentuation oxytone. Nous précisons que nous entendons par mot transparent un mot qui est proche phonétiquement entre l'espagnol et le français, mais 
qui peut différer ou non par l'accentuation (par exemple : mara'ton/ marathon ou 'moto/ moto). Un mot non transparent est un mot sans ressemblance phonétique entre les deux langues (par exemple : za'pato/ chaussure ou pa'red/ mur).

\section{Protocole expérimental}

\subsection{Sujets}

17 Vingt-quatre sujets francophones et huit sujets hispanophones (groupe contrôle) se sont soumis à notre expérience. Nous avons choisi des étudiantes ${ }^{3}$ universitaires en espagnol ${ }^{4}$ avec des niveaux de langue différents correspondants à l'un des trois niveaux de langue décrits par le Cadre européen commun de référence pour les langues (Conseil de l'Europe, 2001) : 8 sujets du niveau A (utilisateurs élémentaires), 8 sujets du niveau B (utilisateurs indépendants) et 8 sujets du niveau C (utilisateurs expérimentés). Dans l'optique d'écarter des interférences et de garantir l'homogénéité des sujets, les francophones qui ont réalisé l'expérience sont d'origine française, obligatoirement monolingues ${ }^{5}$ de langue première française et n'ont pas de connaissance d'une autre langue romane. L'échantillon de sujets de langue première espagnole comprend des personnes de niveau universitaire qui possèdent une prononciation standard de l'espagnol et ne présentent aucune pathologie du langage.

\subsection{Stimuli}

Les 40 stimuli visuels et auditifs sélectionnés sont exactement les mêmes en production et en perception ${ }^{6}$. Les 40 stimuli de l'expérience correspondent à trois critères en fonction de l'aspect à étudier, que ce soit pour la perception ou pour la production, choisis en fonction des variables dépendantes de l'étude. Le premier est le nombre de syllabes: l'échantillon inclut 16 mots de 2 syllabes et 24 mots de 3 syllabes. Le deuxième est le type d'accentuation; il y a trois positions d'accent différentes parmi nos stimuli : 16 mots oxytons, 16 mots paroxytons et 8 mots proparoxytons. Enfin, le dernier critère est la transparence lexicale. Les stimuli sélectionnés pour les expériences sont des mots transparents et non transparents. Il s'agit de mots proches phonétiquement en français et en espagnol et qui peuvent différer ou non dans l'accentuation du mot dans les deux langues (par exemple : « melón / melon », " francés / français », « número / numéro »). Les mots non transparents sont des mots qui n'ont aucune ressemblance phonétique entre les deux langues (par exemple : « azul / bleu », « reloj / montre ", " pájaro / oiseau »). Quatre mots transparents et quatre mots non transparents sont sélectionnés dans notre expérimentation pour chaque type d'accentuation.

Il y a trois critères également qui ont servi à sélectionner les stimuli en fonction des variables contrôlées de l'étude. Tout d'abord, la catégorie grammaticale: nos stimuli appartiennent tous à l'une des deux catégories grammaticales substantifs ou adjectifs, car leur accentuation n'est pas déterminée morphologiquement, comme c'est le cas en espagnol, par exemple, pour les formes verbales (Harris, 1991, p. 174). Deuxièmement, quant à la structure syllabique, la syllabe accentuée du mot a une structure CV pour les mots paroxytons et proparoxytons, et une structure CVC pour les mots oxytons. La structure CV étant la plus courante dans les deux langues (Quilis, 1981, p.335). 
Troisièmement, toutes les phrases possèdent huit syllabes, indépendamment du nombre de syllabes du stimulus concret.

\subsection{Facteurs expérimentaux}

Nous analysons 7680 observations : 32 sujets face à 40 stimuli présentés dans 3 conditions (=3840) dans les deux expériences perception et production. Nous mesurons les résultats obtenus aussi bien en production qu'en perception en fonction de cinq facteurs :

1. Facteur niveau des sujets : les 32 sujets ont été répartis en 4 groupes : niveau A : débutants ; niveau $\mathrm{B}$ : intermédiaires ; niveau $\mathrm{C}$ : expérimentés ; et niveau $\mathrm{D}:$ hispanophones.

2. Facteur type d'intonation : lorsque le stimulus apparait en contexte, il est toujours situé à la fin de la phrase dans le but de considérer l'effet de l'accentuation par rapport à l'intonation. Nous examinons donc trois conditions :

- condition $1:$ mots isolés en intonation descendante

- condition 2 : phrases en intonation descendante

- condition 3 : phrases en intonation montante

3. Facteur type d'accentuation: on étudie les effets liés aux différents types d'accentuation en ce qui concerne la perception et la production de l'accent lexical espagnol selon trois types d'accentuation :

- mots oxytons

- mots paroxytons

- mots proparoxytons

4. Facteur nombre de syllabes:

-2 syllabes

-3 syllabes

5. Facteur transparence lexicale :

- condition 1 : mots transparents

- condition 2 : mots non transparents

\subsection{Procédure de passation des tests}

L'expérience s'est déroulée dans une salle d'enregistrement de parole ${ }^{7}$. Les sujets ont été placés face à un poste informatique et ont passé l'expérience un par un. Ils effectuent d'abord le test de production pour s'assurer qu'une perception préalable n'interfère pas leur production.

\subsubsection{Première partie de l'expérimentation : production}

Les sujets effectuent donc le test de production en prononçant les stimuli à l'aide d'images ${ }^{8}$. Celles-ci sont présentées dans une phrase cadre qu'ils doivent lire. Chaque image représente un mot. Le sujet ne voit pas le mot qu'il doit prononcer afin d'éviter des effets dus à la lecture ou à la visualisation du mot écrit. Il prononce le mot qui correspond à l'image en intonation descendante. Par exemple, il voit une chaussure et prononce le mot «zapato » sans article. Ensuite, il lit la phrase où l'image est insérée et dans laquelle l'image du mot-cible se trouve en position finale, avec l'intonation correspondante, montante ou descendante. 
Figure 1. - Exemple de stimuli : mot représenté par une image inséré dans une phrase avec intonation descendante et dans une phrase avec intonation montante.

\section{Buscamos} este
¿ Buscamos este

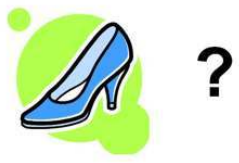
l'expérience.

Les sujets n'ont pas de limite de temps, ils passent d'un stimulus au suivant en cliquant avec la souris. Les stimuli sont présentés à l'écran grâce au logiciel Présentation, qui permet aux images de se succéder les unes après les autres. Ils apparaissent dans un ordre aléatoire qui est le même pour tous les sujets9. En même temps, les réponses des sujets sont enregistrées à l'aide d'un microphone ${ }^{10}$ et un fichier audio .wav est créé (fréquence d'échantillonnage de $44100 \mathrm{~Hz}$ ). Les sujets ont une phase d'entrainement avec la présentation de trois stimuli qu'ils doivent produire comme il est demandé dans

Un évaluateur identifie perceptivement la syllabe sur laquelle les sujets placent l'accent, afin de déterminer si la production des locuteurs est correcte. Dans les cas où il a été difficile de déterminer la syllabe accentuée, comme dans les cas de double accentuation dans un même mot, deux auditeurs supplémentaires natifs, experts linguistes, sont invités à donner leur avis. On adopte ensuite la perception majoritaire.

\subsubsection{Deuxième partie de l'expérimentation : perception}

Dans un second temps, les sujets écoutent à l'aide d'un casque les stimuli (phrases ou mots) à la suite les uns des autres sans possibilité de les réécouter. Ils répondent directement sur l'écran du même poste informatique en cliquant avec la souris sur une grille de réponse ${ }^{11}$. Cette partie de l'expérience se déroule en deux temps :

1. Les sujets écoutent une liste de mots isolés et doivent marquer la syllabe sur laquelle ils perçoivent l'accent ;

2. Ces mots sont intégrés dans de courtes phrases de 8 syllabes. Le mot à percevoir se situe systématiquement en fin de phrase (le dernier mot de la phrase). Les sujets écoutent la liste des phrases présentées aléatoirement et doivent également marquer la syllabe du mot sur laquelle ils perçoivent l'accent.

Avant de réaliser l'expérience, les sujets se soumettent à un pré-test. Ils écoutent trois exemples pour les mots et six exemples pour les phrases (trois mots insérés dans des phrases en intonation montante et descendante), afin de se familiariser avec le type d'expérience. Chaque sujet répond sur une grille de réponse informatisée où il doit sélectionner la syllabe sur laquelle il perçoit l'accent. Dans la grille de réponse pour les mots, ceux-ci sont représentés sous forme de cases correspondant au nombre de syllabes, 
ce qui veut dire que les sujets ne voient à aucun moment le mot écrit (ex. : $\square \square \square$ pour le mot $z a-p a-t o)$.

Dans la grille de réponses pour les phrases, les sujets peuvent lire les phrases qu'ils écoutent à l'exception du mot où ils doivent marquer la perception de l'accent, qui est toujours placé en fin de phrase. Celui-ci est également représenté sous forme de cases correspondant au nombre de syllabes (ex. : Buscamos este $\square \square \square$ pour le mot za-pa-to).

Entre chaque mot et chaque phrase, il y a cinq secondes d'intervalle et l'intervalle est doublé (soit dix secondes) tous les 20 mots. Les stimuli ont été enregistrés par une locutrice native dépositaire d'un accent espagnol standard, originaire de Vitoria et ne présentant pas de troubles du langage. Les stimuli enregistrés étaient homogènes quant au rythme de parole, sans focus emphatique dans la réalisation des accents et présentent une intonation naturelle.

\subsection{Procédure d'analyse de résultats}

Nous mesurons les pourcentages de bonnes réponses (désormais BR). Le logiciel Statistica $8^{12}$ nous permet de réaliser les tests statistiques pertinents (tests de variance ANOVA et tests de Tukey), aussi bien pour les résultats de perception que de production.

\section{Résultats}

\subsection{Effet de la L1}

L'effet de la L1 en perception est significatif: $F(1,30)=12,164, p=0,00153$. En effet, les sujets hispanophones obtiennent $100 \%$ de BR, alors que les francophones atteignent $79,58 \%$ de BR. L'effet de la L1 en production est significatif : $F(1,30)=12,882, p=0,00116$. En effet, les sujets hispanophones obtiennent $100 \%$ de BR, alors que les francophones atteignent $80,69 \%$ de BR. En raison des scores obtenus par les sujets hispanophones, à savoir $100 \%$ de bonnes réponses dans toutes les conditions de l'expérimentation, la présentation des résultats qui suit est restreinte aux trois groupes de sujets francophones.

\subsection{Effets globaux en perception / production}

31 Si nous examinons le traitement de l'accent lexical en comparant les performances obtenues par les sujets entre la perception et la production, toutes conditions confondues, nous constatons que cet effet n'est pas globalement significatif $(F(1,21)=$ $0,13765, p=0,71434)$. Les sujets obtiennent un pourcentage de BR de $79,58 \%$ dans la condition perception et $80,69 \%$ dans la condition production. Toutefois, en regardant plus attentivement certaines interactions, les différences entre la perception et la production s'avèrent significatives. Nous détaillons ensuite ces interactions significatives.

\subsubsection{Effet du facteur niveau des sujets}

Les différences entre la perception et la production de l'accent lexical espagnol en ce qui concerne le facteur niveau des sujets ne sont pas significatives $(F(2,21)=0,99702, p=$ 0,38578). Globalement, les sujets ne le perçoivent pas mieux qu'ils le produisent ou 
inversement, en fonction de leur niveau (A, B ou C). Cependant, l'effet niveau de sujets est significatif dans chacun des deux tests séparément.

En perception, nous observons un effet significatif du niveau des sujets sur le pourcentage de $B R$, toutes autres conditions confondues, $(F(2,21)=14,083, p=0,00013)$. Les sujets qui perçoivent mieux l'accent lexical espagnol sont ceux du niveau $C(93,22 \%$ de BR), suivis des sujets du niveau B ( $81,77 \%$ de BR) puis de ceux du niveau A (63,75 \% de BR).

En production, l'effet du niveau des sujets, toutes conditions confondues, est significatif également: $F(2,21)=36,560, p=0,00000$. La meilleure production de l'accent lexical espagnol est celle des sujets du niveau C ( $98,12 \%$ de BR), suivie de celle des sujets du niveau B ( $76,97 \%$ de BR) et enfin de celle du niveau A (66,97 \% de BR).

\subsubsection{Effet du facteur type d'intonation}

L'effet du facteur type d'intonation dans les conditions mots isolés / phrases en intonation descendante et phrases en intonation montante s'est avéré significatif ( $F$ $(2,42)=27,068, p=0,00000)$. Nous observons que pour les mots présentés isolément avec une intonation descendante et pour les phrases en intonation descendante, les sujets obtiennent des meilleurs résultats dans la condition perception par rapport à la condition production $(86,97 \%$ de BR en perception et $78,85 \%$ de BR en production pour les mots isolés ; $82,39 \%$ de BR en perception et $80,52 \%$ de BR en production pour les phrases en intonation descendante). En revanche, en ce qui concerne les phrases en intonation montante, les sujets obtiennent des pourcentages de BR plus élevés dans la condition production ( $82 \%$ de BR) que dans la condition perception ( $69 \%$ de BR).

Par ailleurs, les résultats révèlent également que la perception des sujets est meilleure pour la condition mots isolés $(86,97 \%$ de BR) que pour la condition mots en contexte, aussi bien pour les phrases avec une intonation descendante (avec un $82,39 \%$ de BR ( $F$ $(1,21)=5,1178, p=0,03441)$ ) que pour les phrases avec une intonation montante (avec un $69,37 \%$ de $B R(F(1,21)=33,972, p=0,00001))$.

Figure 2. - Pourcentages de bonnes réponses de l'effet du facteur mots isolés / phrases en intonation descendante / phrases en intonation montante (PE : perception ; PR : production).

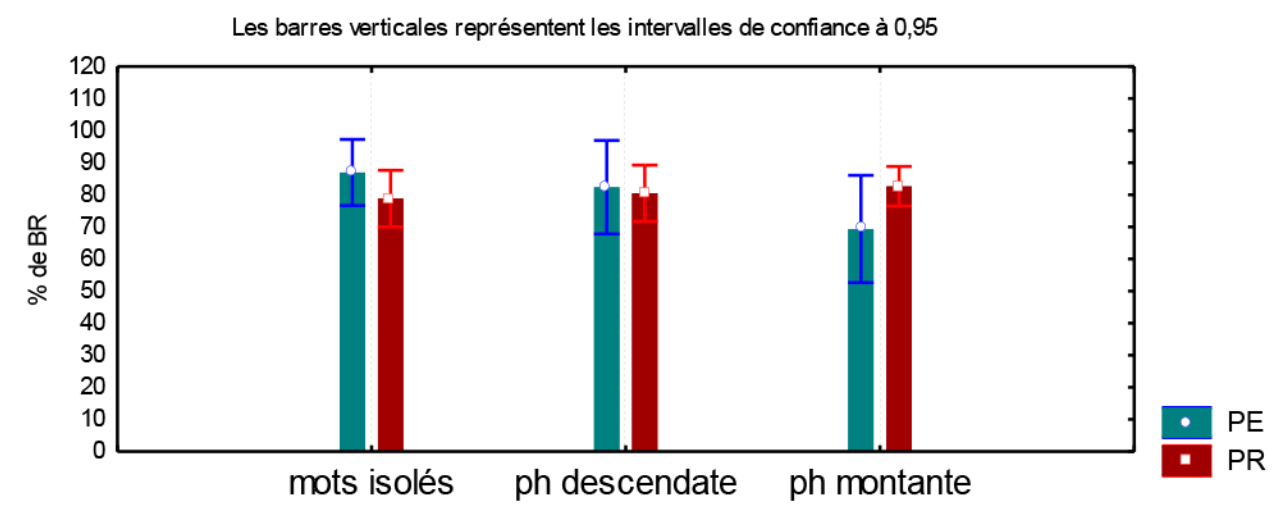

\subsubsection{Effet du facteur nombre de syllabes}

L'effet du nombre de syllabes s'est avéré non significatif entre la condition perception et la condition production : $F(1,21)=0,59192, p=0,45024$. La différence entre la perception 
de mots de deux syllabes et leur production n'est pas significative et la différence entre la perception et la production des mots de trois syllabes non plus.

Toutefois, nous tenons à signaler que l'effet en soi s'est révélé significatif dans les deux conditions séparément. En perception $(F(1,21)=48,266, p=0,00000$, les sujets obtiennent $84,89 \%$ de BR pour les mots de deux syllabes et de $76,04 \%$ de BR pour les mots de trois syllabes.

En production, la différence est également significative $(F(1,21)=23,769, p=0,00008)$ avec $87,32 \%$ de BR pour les mots de deux syllabes et $76,27 \%$ de BR pour les mots de trois syllabes.

\subsubsection{Effet du facteur type d'accentuation}

Nous observons que le comportement des sujets vis-à-vis du type d'accentuation est différent en tâche de perception et en tâche de production : $F(2,42)=9,0229, p=0,00055$. Pour l'accentuation paroxytone, les sujets francophones éprouvent moins de difficultés en production ( $79 \%$ de BR) qu'en perception ( $92 \%$ de BR). En revanche, pour les mots oxytons et proparoxytons, l'accent lexical espagnol est mieux traité en perception qu'en production ( $81 \%$ et $77 \%$ de BR ; $76 \%$ et $63 \%$ de BR respectivement).

Figure 3. - Pourcentages de bonnes réponses de l'effet du facteur type d'accentuation (OX: oxytons ; POX : paroxytons ; PPOX : proparoxytons ; PE : perception ; PR : production).

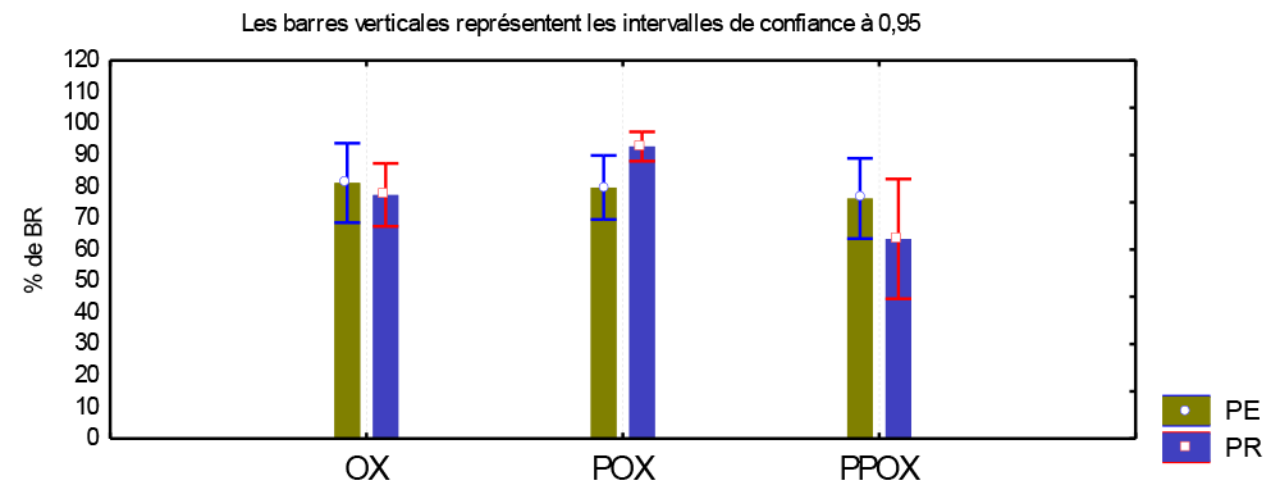

\subsubsection{Interaction entre le facteur niveau des sujets et le facteur type d'accentuation}

41 L'interaction entre le facteur niveau des sujets et le facteur type d'accentuation est significative $(F(4,42)=2,8024, p=0,03774)$. Premièrement, nous pouvons observer dans cette interaction que les sujets des niveaux A et B présentent une distribution des résultats similaire, à savoir ils traitent les mots oxytons et proparoxytons plus difficilement en production qu'en perception (oxytons pour A: $60 \%$ et $74 \%$ de BR; oxytons pour B : $64 \%$ et $85 \%$ de BR; proparoxytons pour A : $38 \%$ et $63 \%$ de BR; proparoxytons pour B : $92 \%$ et $70 \%$ de BR). En revanche, l'accentuation des mots paroxytons est mieux traitée en production (A: $87 \%$ et B : $92 \%$ de BR) qu'en perception ( $A$ : $61,45 \%$ et $B: 83,85 \%$ de $B R$ ). Deuxièmement, les sujets du niveau $C$ réagissent inversement aux sujets des niveaux élémentaire et intermédiaire quant à l'accentuation oxytone et proparoxytone, obtenant des meilleurs scores en production (96\% et $99 \%$ ) qu'en perception ( $91 \%$ et $94 \%$ de BR). Ils obtiennent $93 \%$ et $96 \%$ de BR pour les mots paroxytons en perception et en production respectivement. 
Figure 4. - Pourcentages de bonnes réponses de l'interaction entre le facteur niveau des sujets et le facteur type d'accentuation (OX : oxytons ; POX : paroxytons ; PPOX : proparoxytons ; PE : perception ; PR : production).

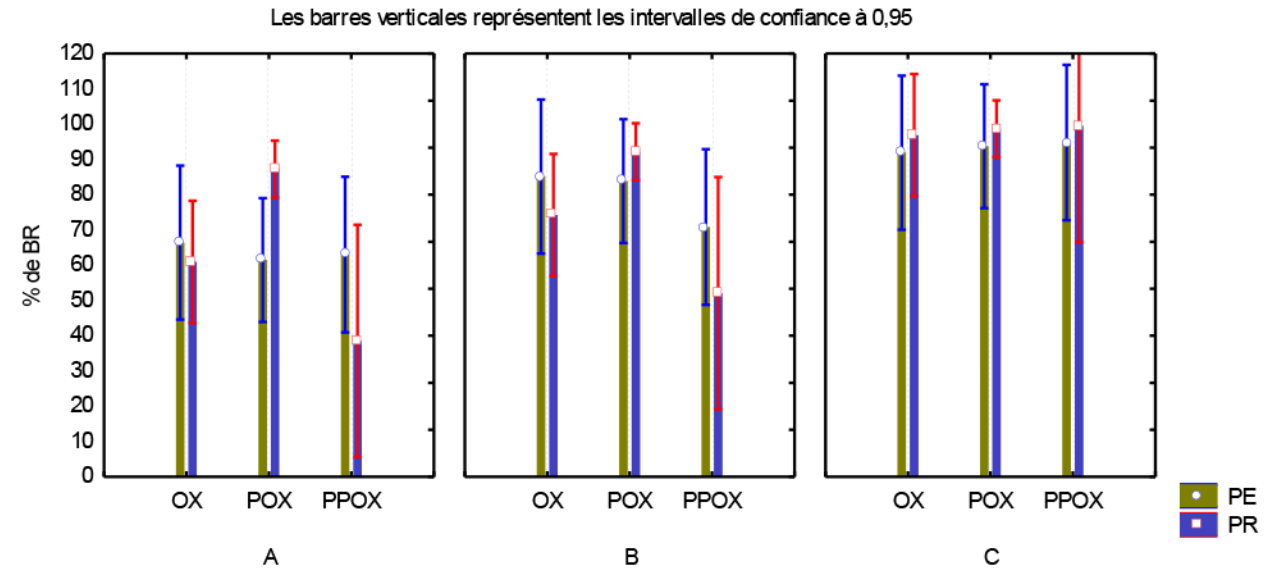

\subsubsection{Effet du facteur transparence lexicale}

Nous avons mis en évidence un effet du facteur transparence lexicale : $F(1,21)=5,5552$, $\mathrm{p}=0,02821$. Néanmoins, on observe qu'en perception, le traitement de l'accent est identique pour les mots transparents et les mots non transparents ( $79 \%$ de BR). En revanche, en production, les pourcentages de BR sont meilleurs pour les mots non transparents ( $83 \%$ de BR) par rapport aux mots transparents ( $77 \%$ de BR).

Figure 5. - Pourcentages de bonnes réponses de l'effet du facteur transparence lexicale (PE : perception ; PR : production).

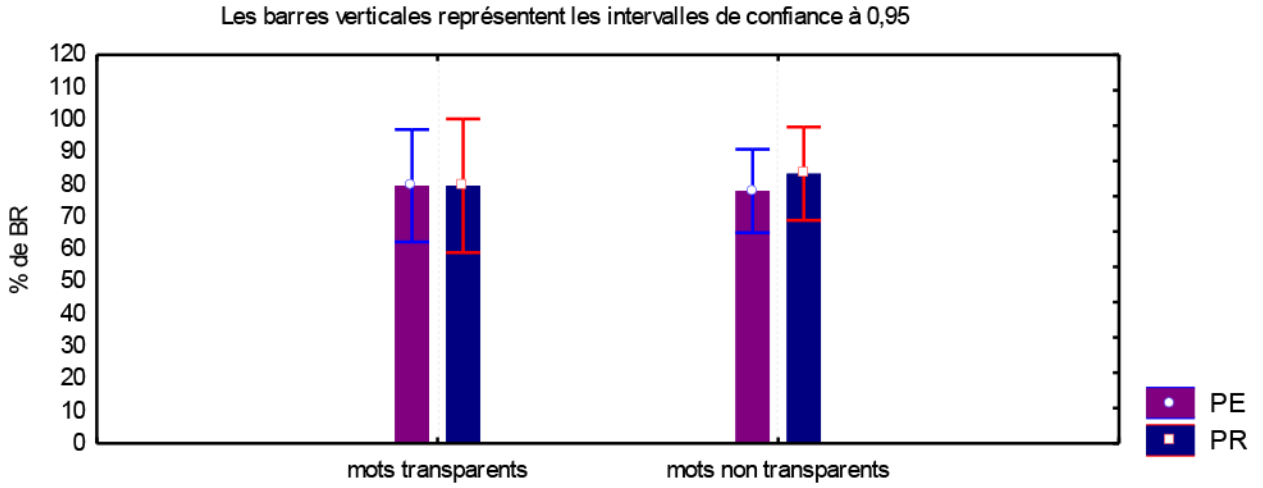

\section{Discussion des résultats}

En premier lieu, au vu des bonnes performances globales obtenues par les francophones lors des deux tâches, nous observons que l'hypothèse de départ qui prévoyait des meilleures performances en perception qu'en production ne s'est pas confirmée. Nous n'avons pas constaté de différences significatives entre la production et la perception concernant le niveau des sujets. Ce résultat semble assez surprenant puisque la tendance à accentuer la dernière syllabe d'un mot ou d'un groupe phonique est un fait que l'on 
remarque régulièrement lors des productions orales des apprenants francophones. Nous pouvons trouver une explication liée à l'évaluation des résultats. Même si l'hypothèse ne se voit pas confirmée, il convient de faire remarquer que, en production, les cas de double accentuation ou triple accentuation pour un même mot peuvent avoir atténué ou influencé les résultats. Dans les cas où l'évaluateur a perçu plusieurs accents, il en a signalé qu'un seul comme correct, et ce fait peut avoir masqué la sensation d'influence de la L1 que l'on a en tant qu'auditeur (enseignant). Pour ce qui est de la perception, la tâche à réaliser et la variabilité phonétique peuvent avoir contribué à la bonne réussite des performances. Dupoux, Pallier, Sebastián-Galles et Mehler (1997, p. 9) et Dupoux, Peperkamp et Sebastián-Galles (2001, p. 1616) ont montré que les sujets francophones, lors d'une tâche de discrimination (AX) avec des stimuli produits par une seule voix présentent peu de difficultés à percevoir l'accent lexical. La surdité accentuelle ${ }^{13}$ dépendrait donc, d'une part, de la charge cognitive requise par la tâche effectuée (tâche $\mathrm{AX}$ ou $\mathrm{ABX}$ ou dans notre cas tâche d'identification) et, d'autre part, de la variabilité phonétique des stimuli présentés aux sujets.

Les résultats de cette étude font supposer que l'acquisition phonique d'un apprenant évolue parallèlement au niveau de la perception et de la production, même si tous les aspects ne sont pas acquis ou traités au même moment et que l'on observe des effets différents selon le stade de l'interlangue de l'apprenant.

Quant à l'effet de niveau, les résultats ne mettent pas en évidence de différences significatives entre la perception et la production en fonction du niveau de langue des sujets. Ce résultat est en relation avec l'effet non significatif de la L1 sur cette comparaison (perception vs production). Comme nous l'avons indiqué, au fur et à mesure que l'expertise des sujets s'améliore, les résultats sont aussi meilleurs, ce qui semble assez logique. Ceci va dans le même sens que d'autres études en perception (Schwab \& Llisterri, 2010, p. 325) et en production (Lafrance, 2001, p. 111).

L'analyse de l'effet du type d'intonation permet d'établir que l'intonation descendante se révèle un contexte facilitateur pour la perception de l'accent lexical (meilleures performances que pour la production), alors que l'intonation montante est davantage facilitante pour la production de l'accent que pour sa perception. Nous pensons qu'en test de perception, les sujets ont eu plus de difficultés avec l'intonation montante, car ils sont possiblement perturbés par l'intonème montant de ce type de phrases et ils ont donc plus de difficultés à différencier la marque de l'accentuation et celle de l'intonation. Tout porte à croire que les sujets francophones auraient recours à des indices acoustiques qui ne font pas partie de leur système phonologique pour d'identifier la syllabe accentuée comme le suggèrent les travaux de Dupoux, Pallier, Sebastián-Galles et Mehler $(1997$, p. 9) et de Schwab et Llisterri (2010, p. 325). L'étude de Schwab et Dellwo (2017, p. 2426-2427) présente des résultats qui vont dans le même sens. La variabilité phonétique nuit à la capacité de détection de l'accent et les différentes formes de variabilité phonétique (locuteur, intonation) n'ont pas les mêmes effets sur des sujets avec une L1 d'accent fixe ou libre. La variabilité d'intonation dans leur étude a eu un impact négatif plus important sur la capacité de détection de l'accent pour les sujets francophones que pour les autres groupes de sujets avec un L1 d'accent libre (espagnols et allemands). Notre étude montre également que la variabilité d'intonation a des effets sur la perception de l'accent lexical de l'espagnol (meilleur en intonation descendante que montante), mais aussi sur la production (meilleur en intonation montante que descendante). Ce résultat nous parait particulièrement intéressant d'un point de vue didactique pour présenter en cours des 
items placés aussi en intonation montante pour travailler la perception de l'accent ${ }^{14} \mathrm{et}$, de la même manière, introduire une variabilité d'intonation afin de travailler la production de l'accent lexical espagnol.

Concernant l'effet du nombre de syllabes, nous avons constaté que le traitement de l'accent est plus difficile dans les deux tâches avec les mots de trois syllabes qu'avec les mots de deux syllabes. Les mots de deux syllabes peuvent être considérés comme des éléments facilitateurs pour la perception et la production de l'accent. La tâche cognitive est plus difficile en fonction du nombre de syllabes à traiter.

Les résultats concernant le type d'accentuation montrent qu'il y a effet significatif selon la tâche. Les sujets traitent mieux les mots oxytons et proparoxytons en tâche de perception qu'en production. En revanche, les mots paroxytons sont mieux traités en production qu'en perception. Concernant la production, l'accentuation des mots paroxytons est celle pour laquelle les sujets obtiennent des meilleures performances et qui est acquise le plus rapidement. Cela peut s'expliquer en partie par le fait que, comme l'accentuation paroxytone est la plus fréquente en espagnol, les francophones tendent à la réaliser à l'oral de manière systématique (parfois à la surgénéraliser) et éprouvent moins de difficultés. En termes de difficultés, l'accentuation oxytone est celle que les francophones acquièrent en deuxième lieu. Finalement, la production de l'accent lexical des mots proparoxytons est celle qui s'avère la plus difficile.

Lorsqu'on regarde de près l'interaction des facteurs niveau des sujets et type d'accentuation, elle montre que les différents patrons d'accentuation ne sont pas acquis de la même manière. En production, les bonnes performances des mots paroxytons y compris par les sujets débutants reflètent qu'il s'agit du patron acquis le plus rapidement. Cependant, les performances du même niveau sont beaucoup plus faibles pour la perception et ne montrent pas de différences avec les autres types d'accentuation. Si l'on observe les performances du niveau $\mathrm{B}$, nous constatons que les différences sont les mêmes quant aux patrons accentuels, mais beaucoup plus réduites quant aux pourcentages de BR. Enfin lorsque les sujets atteignent un niveau de langue élevé, les performances sont homogènes aussi bien pour la production que pour la perception, prouvant que les patrons accentuels ont été acquis. Cela montre, d'une part, que l'apprentissage de l'accentuation de la L2 a évolué et, d'autre part, qu'elle ne suit pas la même évolution pour la perception que pour la production.

50 Finalement, les résultats révèlent que la transparence lexicale n'a pas d'impact sur le traitement de l'accent en tâche de perception. Toutefois, les résultats obtenus laissent apparaitre une différence de traitement en production en fonction de la transparence lexicale. Les sujets obtiennent des performances supérieures dans la condition mots non transparents que dans la condition mots transparents. Il semblerait que la similarité phonétique entre les mots en français et les mots en espagnol pose des difficultés supplémentaires concernant l'accentuation correcte des mots en production. La similarité s'avère un élément synonyme de difficulté et non de facilité.

\section{Conclusions}

51 La relation entre perception et production reste toujours une des grandes questions à éclaircir et à approfondir. Comme le souligne Llisterri (1995, p. 98), la capacité perceptive ne garantit pas nécessairement une capacité de production ou vice-versa. Selon Blanco 
Canales (2016, p.10), les processus de perception et de production phonique sont profondément reliés, ce qui a porté à croire qu'ils évoluent à l'unisson puisqu'ils sont dépendants l'un de l'autre. Les résultats de Blanco Canales (2016, p. 4-11) ${ }^{15}$ suggèrent qu'il existe des similitudes importantes entre les processus d'acquisition de la composante phonique de la L1 et de la L2 et que, dans les deux cas, une perception efficace précède une production efficace. Les résultats de nos expériences montrent que pour ce qui est de l'accentuation, les cas de bonne perception n'aboutissent pas obligatoirement à une production correcte et réciproquement. Il est vrai que, dans le domaine de l'enseignement des langues étrangères, il existe une tendance à croire assez répandue qu'une bonne production est impossible sans la perception préalable, mais aussi qu'une mauvaise perception est la cause des productions incorrectes. La perception et la production sont des phénomènes reliés, certes, mais ils ne font pas forcément un chemin parallèle pendant l'acquisition de la prononciation d'une L2 comme nos résultats ont pu le montrer en ce qui concerne, par exemple, le type d'accentuation en fonction du niveau de langue, le type d'intonation ou encore la transparence lexicale.

La question de la relation entre perception et production mérite d'être posée aussi au niveau de l'enseignement afin d'affiner les choix dans les propositions didactiques et, dans ce sens, cette étude apporte des réponses concrètes qui peuvent guider les choix d'enseignement de l'accentuation en cours. Dans une perspective didactique, nous envisageons les résultats comme contextes facilitant ou non le travail d'accentuation en cours, en tout cas, comme des éléments à ne pas négliger et à considérer dans la réflexion didactique. L'enseignant peut en tenir compte pour les programmations ou pour la création de matériel. On peut adapter les difficultés en fonction du niveau, insistant, par exemple, sur l'accentuation oxytone et proparoxytone dès les niveaux débutant et intermédiaire. On peut avoir à l'esprit que l'intonation facilitera ou rendra difficile certains types d'accentuation ou encore que les similarités entre les deux langues (accentuation oxytone ou mots transparents) peuvent, bien au contraire, être des éléments de difficulté.

Cette étude ne prétend pas, bien évidemment, présenter un inventaire exhaustif des difficultés que l'on peut rencontrer. Les résultats que nous avons mis en évidence permettent d'avoir une idée plus précise sur les phénomènes de perception et de production de l'accent lexical de l'espagnol par des apprenants francophones en apportant quelques réponses issues d'une étude expérimentale à de possibles questions d'enseignement. Dans tous les cas, d'autres études expérimentales supplémentaires seraient nécessaires pour apporter plus de précisions.

\section{BIBLIOGRAPHIE}

Blanco CANALES, Ana. (2016). La influencia de la lengua materna en la percepción fónica del español/L2. Loquens, 3(1). <http://dx.doi.org/10.3989/loquens.2016.028>.

CONSEIL DE L'EUROPE. (2001). Cadre européen commun de référence pour les langues. Strasbourg : Didier. 
CORTÉS MORENO, Maximiano. (2002). Didáctica de la prosodia del español: la acentuación y la entonación. Madrid : Edinumen.

DI CRISTO, Albert. (1981). Vers une modélisation de l'accentuation du français. Aix-en-Provence : Éditions de l'Université de Provence.

Dupoux, Emmanuel, PAllier, Christophe, Sebastián-GAlles, Nuria, \& MeHLER, Jacques. (1997). A Destressing "Deafness" in French? Journal of Memory and Language, 36(3), 406-421.

Dupoux, Emmanuel, Peperkamp, Sharon, \& Sebastián-Gallés, Nuria. (2001). A Robust Method to Study Stress “Deafness". Journal of the Acoustical Society of America, 110(3), 1606-1618.

Frost, Dan. (2010). La surdité accentuelle : d'où vient-elle et comment la guérir ? Cahiers de l'APLIUT, XXIX(2). <https://doi.org/10.4000/apliut.684>.

GIL FERNÁNDEZ, Juana. (2007). Fonética para profesores de español: de la teoría a la práctica. Madrid : Arco Libros.

HARRIS, James W. (1991). La estructura silábica y el acento en español. Madrid : Visor Distribuciones. IRUELA, Agustin. (2004). Adquisición y enseñanza de la pronunciación en lenguas extranjeras (Thèse de doctorat). Universidad de Barcelona, Barcelone.

HERMENT, Sophie. (2018). Apprentissage et enseignement de la prosodie : l'importance de la visualisation. Revue française de linguistique appliquée, XXIII(1), 73-88.

HERRY-BENIT, Nadine \& PILlot-Loiseau, Claire. (2012, juin). Enseignement et évaluation de la prononciation de l'anglais et du français langues étrangères. Communication présentée au colloque « Langages, cultures, sociétés : interrogations didactiques. Colloque Diltec», Paris.

HidAlgo NAVARRo, Antonio \& Quilis MERIN, Mercedes. (2004). Fonética y fonología españolas. Valence : Tirant lo blanch.

LAFRANCE, Jenny. (2001). La production de l'accent espagnol par des francophones. Dans Actes du Colloque des étudiants en sciences du langage de l'université du Québec (CESLa), 1, 105-118. Disponible en ligne sur <www.er.uqam.ca/nobel/scilang/> (consulté le 8 mars 2019).

LÉon, Pierre. (1992). Phonétisme et prononciations du français. Paris : Nathan.

LLISTERRI, Joaquim. (1995). Relationships between Speech Production and Speech Perception in a Second Language. Dans Actes du $13^{e}$ International Congress of Phonetic Sciences (ICPhS), 4, 92-99. Disponible en ligne sur <http://liceu.uab.es/ joaquim/publicacions/ LListerri_95_L2_Production_Perception.pdf> (consulté le 8 mars 2019).

LHOTE, Élisabeth. (1995). Enseigner l'oral en interaction : percevoir, écouter, comprendre. Paris : Hachette.

Quilis, Antonio. (1981). Fonética acústica de la lengua española. Madrid : Gredos.

Quilis, Antonio. (1993). Tratado de fonología y fonética españolas. Madrid : Gredos.

Mora, Elsa, CourToIs, Fabienne \& CAVÉ, Christian. (1997). Étude comparative de la perception par des sujets hispanophones et francophones de l'accent lexical en espagnol. Revue Parole, 1, 5-85.

Muñoz García, Margarita, PAnissal, Nathalie, Billières, Michel \& BAquÉ, Lorraine. (2009). ¿La metáfora de la criba fonológica se puede aplicar a la percepción del acento léxico español? Estudio experimental con estudiantes francófonos. Dans C. M. Bretones Callejas et coll. (dir.), Applied Linguistics Now: Understanding Language and Mind = La Lingüística aplicada actual: comprendiendo el lenguaje y la mente (p. 489-500). Almería : Universidad de Almería. 
MUÑOZ GARCÍA, Margarita. (2010). La perception et la production de l'accent lexical de l'espagnol par des francophones : aspects phonétiques et psycholinguistiques (Thèse de doctorat). Université de Toulouse, Toulouse.

RASIER, Laurent. (2003). Le système accentuel de l'interlangue d'apprenants francophones du néerlandais. Dans A. Mettouchi \& G. Ferré (dir.), Actes du colloque international « Interfaces Prosodiques » (p. 79-84). Nantes : A.A.I., Université de Nantes. Disponible en ligne sur <http:// llacan.vjf.cnrs.fr/pers/mettouchi/ip2003/actes-ip2003.pdf> (consulté le 8 mars 19).

RASIER, Laurent \& HILIGSMANN, Philippe. (2007). Prosodic Transfert from L1 to L2. Theoretical and Methodological issues. Nouveaux cahiers de linguistique française, 28, 41-66.

SCHWAB, Sandra \& LLISTERRI, Joaquim. (2010). La perception de l'accent lexical espagnol par des apprenants francophones. Dans L. Baqué \& M. Estrada (dir.), La langue et l'être communiquant. Hommage à Julio Murillo (p. 311-28). Mons : Centre international de phonétique appliquée.

SCHWAB, Sandra \& Dellwo, Voler. (2017). Intonation and Talker Variability in the Discrimination of Spanish Lexical Stress Contrasts by Spanish, German and French Listeners. The Journal of the Acoustical Society of America, 142, 2419-2429. <https://doi.org/10.1121/1.5008849>.

SPALACCI, Mireille. (2014). Médiatisation orale de l'enseignement de la prononciation. Synergies Espagne, 7, 175-187.

Sebastián-Gallés, Nuria, Bosch, Laura \& Costa, Albert. (1999). La percepción del habla. Dans M. de Vega \& F. Cuetos (dir.), Psicolingüística del español (p. 53-88). Madrid : Editorial Trotta.

\section{ANNEXES}

ANNEXE $n^{\circ} 1$ : liste de stimuli

\begin{tabular}{|l|l|l|l|l|l|}
\hline & OX2 & POX2 & OX3 & POX3 & PPOX3 \\
\hline \multirow{4}{*}{ Mots non transparents } & MZUL & MESA & CORAZON & MANZANA & SABADO \\
& PINTOR & LECHE & VEINTIDOS & CABEZA & DECIMO \\
& PARED & GATO & CALCETIN & CAMISA & PAJARO \\
\hline \multirow{2}{*}{ Mots transparents } & MRANCES & MOTO & ASCENSOR & ESPAÑA & PUBLICO \\
& MELON & DUCHA & HOSPITAL & TOMATE & CAMARA \\
& PARIS & ROSA & JAPONES & BOTELLA & NUMERO \\
\hline
\end{tabular}

\section{$\operatorname{ANNEXE~} n^{\circ} 2$ : liste des stimuli en contexte}

L'ordre de stimuli (phrases en intonation montante ou descendante) est présenté de façon aléatoire. Nous présentons la liste des stimuli insérés dans les phrases sans son contour intonatif. 


\begin{tabular}{|l|l|}
\hline Alejandro es un pintor & José toca la guitarra \\
Alfonso bebe la leche & Lo cocino con tomate \\
Aquel pantalón es rosa & Lo filmo con la cámara \\
Aquí cogen el número & Lo ponemos en la mesa \\
Bebes zumo de manzana & Me lo dice de corazón \\
Buscamos este zapato & Mi piso es el décimo \\
Cantó para su público & No te gusta este gato \\
Comes carne con tenedor & No trabajas el sábado \\
David escucha música & Nos ponemos la camisa \\
El loro es un pájaro & Pablo duerme en su cama \\
El mar es de color azul & Quiere conducir la moto \\
El señor Cruz es médico & Subimos con el ascensor \\
Ellos corren un maratón & Te lavas en una ducha \\
En clase somos veintidós & Teresa es una mujer \\
En Francia se habla francés & Tiene dolor de cabeza \\
En Japón hablan japonés & Trabajan en un hospital \\
En verano como melón & Tú te pones el calcetín \\
Esta capital es París & Vamos a coger un taxi \\
Este vestido es marrón & Van de viaje a España \\
Hay vino en la botella & Antonio pinta la pared \\
\hline
\end{tabular}

\section{NOTES}

1. Nous avons opté par un test d'identification de la syllabe accentuée d'un mot lors du test de perception afin de pouvoir évaluer et comparer les mêmes items en tâche de perception et en tâche de production. De ce fait, les mêmes mots avec leurs syllabes accentuées correspondantes sont prononcés dans les mêmes conditions en tâche de production.

2. Dans le cas de l'étude mentionnée, l'intonation montante a été évaluée à partir de stimuli insérés à la fin des phrases interrogatives avec une intonation montante finale. Nous avons repris le même protocole pour cette expérience.

3. Nous avons choisi des sujets exclusivement féminins afin d'éviter l'effet des différences acoustiques entre les voix d'hommes et les voix de femmes, car nous avons également réalisé des analyses acoustiques des productions orales qui ne font pas l'objet de cet article.

4. Étudiantes en espagnol de l'université Toulouse 2.

5. Par monolingue nous entendons des francophones ayant comme L1 le français. Les autres langues de connaissance sont des L2.

6. Annexe $n^{\circ} 1$ : liste de stimuli.

7. Laboratoire Jacques Lordat - OCTOGONE - EA 4156, Université Toulouse 2.

8. Les images sont issues du programme ClipArt.

9. Annexe $\mathrm{n}^{\circ} 2$ : liste de stimuli en contexte. 
10. Référence: marque: BST; modèle: MC-504; émetteur: BP-193-UHF Wireless System. Récepteur: marque: BST ; modèle: HF ; récepteur: UDR-193. Carte son: marque : MOTU; modèle : 896 HD.

11. Nous avons créé un formulaire de saisie informatisé permettant aux sujets de saisir les réponses, puis de les stocker sur l'ordinateur dans un fichier au format .txt (technologie utilisée : HTML, Java Script).

12. Statsoft France (2008). Statistica (logiciel d'analyse de données), version 8.0, <www.statsoft.fr>. 13. Nous dénommions « surdité accentuelle » la surdité phonologique propre à l'accentuation.

14. Et d'autres types d'intonation (emphatique, suspendue et autres qui ne font pas partie de cette étude) bien entendu, pas seulement l'intonation descendante comme c'est souvent le cas.

15. Résultats d'une étude de perception phonique de l'espagnol/L2 par des locuteurs de 16 pays qui se sont soumis à des tests de perception afin d'étudier l'influence de la L1 sur la perception de l'espagnol en fonction de leur L1.

\section{RÉSUMÉS}

Cette recherche a pour objet l'étude de la perception et de la production de l'accent lexical espagnol par des francophones. Les systèmes accentuels des deux langues sont différents. Le français est une langue à accent fixe, tandis que l'espagnol est une langue à accent libre avec plusieurs types d'accentuation possibles. Ainsi, nous avons adopté une approche expérimentale afin de tester l'influence de la langue première (L1) des francophones sur la perception et la production de l'accent lexical espagnol. Les résultats montrent que, pour ce qui est de l'accentuation, les cas de bonne perception n'aboutissent pas nécessairement à une bonne production et réciproquement. D'un point de vue didactique, les résultats obtenus ont permis d'avoir des renseignements plus amples sur l'interlangue des apprenants francophones. Cette étude a permis de mettre en évidence certains éléments ou conditions qui s'avèrent être des contextes difficiles pour l'apprentissage de l'accentuation et font émerger des contextes facilitateurs pour certains aspects spécifiques de l'accentuation.

This study aims to assess how French native speakers perceive and produce Spanish lexical stress. The stress systems of these two languages are different: while the French is a fixed lexical stress language, Spanish language is characterized by a free lexical stress system. Hence, an experimental approach was undertaken in order to examine the influence of L1 French on the perception and production of lexical stress in L2 Spanish. Subjects' performance was examined by comparing the processing of stimuli during perception and production tasks. It appeared that the subjects' performance improved during the acquisition stages in both perception and production tasks. Conversely, different stress placement was not processed equally during both tasks. From a didactic point of view, the results showed that the learners' interlanguage develops at several levels according to different stages of L2 stress acquisition. The study made it possible to highlight elements and conditions that create hindering contexts for stress acquisition, and others that promote the learning of specific aspects of stress. 
INDEX

Mots-clés : perception, production, accent lexical, espagnol, français, apprentissage de la prononciation

Keywords : perception, production, lexical stress, Spanish language, French language, pronunciation learning

\section{AUTEUR}

MARGARITA MUÑOZ GARCÍA

LIDILE (Linguistique, Ingénierie, Didactique des langues), Université Rennes 2 